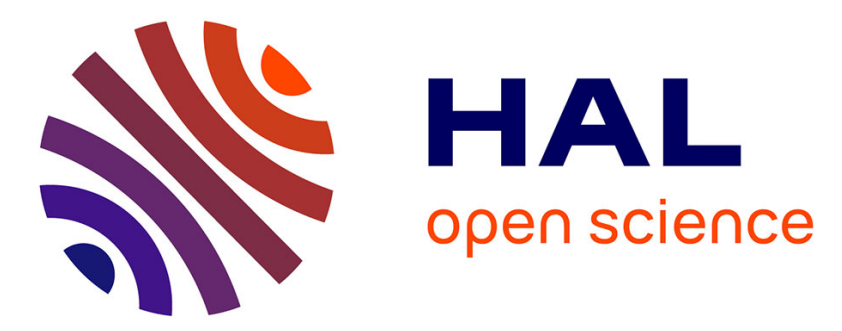

\title{
Photoinduced dynamics of ethene in the N, V, and Z valence states: A six-dimensional nonadiabatic quantum dynamics investigation
}

Alexandra Viel, Robert P. Krawczyk, Uwe Manthe, Wolfgang Domcke

\section{To cite this version:}

Alexandra Viel, Robert P. Krawczyk, Uwe Manthe, Wolfgang Domcke. Photoinduced dynamics of ethene in the $\mathrm{N}, \mathrm{V}$, and $\mathrm{Z}$ valence states: A six-dimensional nonadiabatic quantum dynamics investigation. Journal of Chemical Physics, 2004, 120 (23), pp.11000-11010. 10.1063/1.1740696 . hal01118367

\section{HAL Id: hal-01118367 \\ https://hal.science/hal-01118367}

Submitted on 10 Jul 2017

HAL is a multi-disciplinary open access archive for the deposit and dissemination of scientific research documents, whether they are published or not. The documents may come from teaching and research institutions in France or abroad, or from public or private research centers.
L'archive ouverte pluridisciplinaire HAL, est destinée au dépôt et à la diffusion de documents scientifiques de niveau recherche, publiés ou non, émanant des établissements d'enseignement et de recherche français ou étrangers, des laboratoires publics ou privés. 


\title{
Photoinduced dynamics of ethene in the $N, V$, and $Z$ valence states: A six-dimensional nonadiabatic quantum dynamics investigation
}

\author{
Alexandra Viel, ${ }^{\text {a) }}$ Robert P. Krawczyk, ${ }^{\text {b) }}$ Uwe Manthe, ${ }^{\text {c) }}$ and Wolfgang Domcke \\ Department of Chemistry, Technical University of Munich, D-85747 Garching, Germany
}

(Received 3 March 2004; accepted 23 March 2004)

\begin{abstract}
The photoinduced dynamics of ethene following $\pi \rightarrow \pi^{*}$ excitation is investigated by quantum wave-packet dynamics on three coupled six-dimensional diabatic potential-energy surfaces representing the $N, V$, and $Z$ valence states, which have been developed previously [J. Chem. Phys. 119, 1397 (2003)]. The C-C stretching and torsion, as well as the pyramidalization and scissoring of both $\mathrm{CH}_{2}$ groups are included in this description. The wave-packet calculations have been performed using the multiconfigurational time-dependent Hartree method for a time period up to 100 fs. While a small amount of population transfer to the electronic ground state is found within this period, the overall population decay time of the $V$ state is found to exceed the 100 fs range significantly. The autocorrelation function of the wave packet and the stationary absorption spectrum of the $V$ state also have been calculated. It is found that both the torsional mode as well as the $\mathrm{C}-\mathrm{C}$ stretching mode contribute to the very extended vibrational structure of the absorption spectrum, and that both modes are strongly coupled. At least on the present ab initio surface of limited dimensionality, the speed of pyramidalization of $90^{\circ}$ twisted ethene appears as the bottleneck for the ultrafast radiationless decay of the $V$ state. (C) 2004 American Institute of Physics.
\end{abstract} [DOI: 10.1063/1.1740696]

\section{INTRODUCTION}

The dynamics induced by $\pi \rightarrow \pi^{*}$ excitation of the carbon-carbon double bond is one of the most fundamental phenomena of organic photochemistry. ${ }^{1}$ It is involved in the photophysics and photochemistry of small polyenes (e.g., butadiene), of diphenylpolyenes (e.g., stilbene), and of large polyenes of photobiological relevance (e.g., retinal, cartenoids), to mention just a few.

Despite decades of research, the UV spectroscopy and photochemistry of even the smallest system with a CC double bond, ethene, are not yet fully understood. The assignment of the UV transitions to electronic states and the interpretation of the vibronic structure of the absorption spectrum of ethene are still a matter of debate, see Refs. 2-5 and references therein. At least half a dozen of different assignments of the vibrational structure of the ${ }^{1} \pi \pi^{*}$ valence transition have been proposed. ${ }^{3,5}$

Following the pioneering work of Mulliken, ${ }^{2,6}$ selected features of the excited-state potential-energy (PE) surfaces of ethene have been investigated by many researchers with electronic-structure methods of increasing sophistication. For an overview of the extensive computational work on the vertical electronic spectrum of ethene, we refer to Refs. 7 and 8 and the references therein. The first accurate calculations of the torsional PE functions of the three valence electronic states $N$ (for normal, that is, ground state), $V$ (for valence),

\footnotetext{
a) Present address: LPQ_IRSAMC, University P. Sabatier, 118 route de Narbonne, F-31062 Toulouse, France.

b) Present address: Dept. of Chemistry, University of Auckland, Private Bag 92019, Auckland, New Zealand.

${ }^{c}$ Present address: Dept. of Chemistry, Theoretical Chemistry, Universitätsstraße 25, D-33615 Bielefeld, Germany.
}

and $Z$ (for zwitterionic) have been performed with the MRD-CI method by Buenker et al. ${ }^{9}$ and Persico and Bonačić-Koutecký. ${ }^{10}$ The so-called sudden-polarization effect, arising from the near degeneracy of $V$ and $Z$ in $90^{\circ}$ twisted ethene, has been investigated in Refs. 9, 11, and 12. Three-dimensional PE surfaces (torsion, CC stretch, symmetric scissors) of the valence states and a selected Rydberg state have been obtained by Groenenboom at the multireference CEPA level. ${ }^{13}$ Additional nuclear degrees of freedom (hydrogen migration and hydrogen detachment) have been considered by Ohmine ${ }^{14}$ and Evleth and Sevin. ${ }^{15}$ Freund and Klessinger ${ }^{16}$ have determined the geometry of the minimum of the seam of intersection of the $S_{1}$ and $S_{0}$ surfaces at the CASSCF level. The conical intersections between the $N, V$, and $Z$ states have been characterized in more detail by BenNun and Martínez ${ }^{17}$ and Molina et al. ${ }^{18}$ at the MRCI and CASPT2 levels, respectively.

Recently, the present research group has developed a sixdimensional $a b$ initio PE surface of the three valence states of ethene ${ }^{19}$ (henceforth referred as paper I). The torsion, the CC stretch coordinate, the left and right pyramidalizations, and the left and right scissors angles have been considered, while $\mathrm{CH}$ stretch coordinates and left and right rocking angles have been kept frozen (at their ground-state equilibrium values). The CASSCF (complete-active-space selfconsistent-field)/CASPT2 (second-order perturbation theory with respect to the CASSCF reference) electronic structure model $^{20,21}$ has been employed, with a cc-pVTZ basis set. ${ }^{22}$ The diabatization of the $V-Z$ and $Z-N$ conical intersections has been achieved in the framework of a Hückel-type model. The parameters of the analytic diabatic PE functions have been determined by a least-squares fit of the eigenvalues of 
the diabatic $3 \times 3$ PE matrix to the ab initio data for onedimensional and two-dimensional cuts of the adiabatic PE surfaces. ${ }^{19}$

In the present work, the valence-state spectroscopy and photochemistry of ethene has been explored via timedependent quantum wave-packet calculations on the nonadiabatically coupled 6D PE surfaces of paper I. The multiconfigurational time-dependent Hartree (MCTDH) methodology $23-25$ is used. It has been shown previously that the MCTDH method is a powerful tool, especially for the fully microscopic description of radiationless decay processes via conical intersections. ${ }^{26-29}$

Ben-Nun and Martínez ${ }^{17,30,31}$ and Granucci et al. ${ }^{32}$ recently have performed computational studies of the photochemistry of ethene using so-called "on-the-fly" (or "direct dynamics") methods, whereby the PE surfaces and the nonadiabatic couplings are computed when needed along classical or Gaussian wave-packet trajectories. The strengths and limitations of the quantum-dynamics approach based on analytical PE surfaces and the direct-dynamics approach are largely complementary. While the direct-dynamics calculations are full dimensional, they are restricted (owing to computingtime limitations) to rather crude electronic-structure models and involve either an approximate treatment of the nonadiabatic dynamics (surface-hopping ${ }^{33}$ or Ehrenfest mean-field ${ }^{34}$ dynamics) or severe approximations in the implementation of more accurate methods (e.g., the multiple-spawning method $\left.^{35}\right)$. In the a priori construction of PE surfaces, on the other hand, a considerably more sophisticated electronicstructure theory (basis set, active space, inclusion of nondynamical electronic correlation) can be employed, but the surfaces are necessarily of reduced dimensionality for a sixatom system like ethene and are often incomplete with respect to the nonseparability of coordinates. The comparison of state-of-the-art implementations of the two competing concepts in contemporary theoretical photochemistry should be of interest beyond the specific case of the ethene molecule.

\section{POTENTIAL-ENERGY SURFACES AND KINETIC- ENERGY OPERATOR}

In paper I, a three-sheeted six-dimensional potentialenergy surface for the $N, V$, and $Z$ valence states has been developed. The analytical representation of the diabatic potential-energy matrix elements consists of restricted twobody expansions including six internal nuclear coordinates. In this expansion, the torsional coordinate is coupled pairwise to the other five coordinates. The torsional angle $(\varphi)$ is defined as the angle between the two vectors connecting the hydrogen atoms of the $\mathrm{CH}_{2}$ groups. The other five coordinates retained are the $\mathrm{C}-\mathrm{C}$ distance $(r)$, the two one-sided pyramidalizations $\left(\vartheta_{r}, \vartheta_{l}\right)$, defined as the angle of the vector from the carbon atom to the center of the two hydrogen atoms with the axis through the two carbon atoms, and finally the two scissors angles $\left(\alpha_{r}, \alpha_{l}\right)$ of the two $\mathrm{CH}_{2}$ groups. The parameters of these expansions have be determined by a least-squares fit to accurate CASPT2 ab initio data. $^{19}$
In this model, the $V$ and $Z$ (and the $V$ and $N$ ) diabatic states are coupled linearly by the antisymmetric scissoring mode and quadratically by the pyramidalizations. As a function of pyramidalization of perpendicular ethylene, the model describes the intersections of the diabatic $N$ and $Z$ states, which are converted into conical intersections by displacements in the torsional mode. The involvement of the scissoring modes in the excited dynamics was mentioned in the literature ${ }^{36,37}$ but only very recently its role as a nonadiabatic coupling mode has been pointed out. ${ }^{12}$ The analytic 6D PE surface contains several conical intersections, which are assumed to trigger an ultrafast internal-conversion process from the photoexcited $V$ state to the ground state. ${ }^{16,32,35,38}$ The first type of conical intersection, involving the $V$ and $Z$ excited states, occurs along the torsional mode for $\varphi \simeq 82^{\circ}$. Another conical intersection between the adiabatic ground state and the first excited state takes places along pyramidalization of one of the $\mathrm{CH}_{2}$ group starting from the $D_{2 d}$ geometry $\left(\varphi=90^{\circ}\right)$.

The kinetic-energy term corresponding to the six internal coordinates $q_{i} \in\left\{\varphi, \vartheta_{r}, \vartheta_{l}, \alpha_{r}, \alpha_{l}, r\right\}$ has been approximated in the following way. First the $6 \times 6$ matrix

$$
G_{q_{i} q_{j}}^{-1}=\sum_{n=1}^{18} \frac{\partial X_{n}}{\partial q_{i}} \frac{\partial X_{n}}{\partial q_{j}}
$$

where the $X_{n}$ are Cartesian coordinates of the six atoms of the molecule, has been analytically computed. This matrix is block diagonal with the following zero elements:

$$
\begin{aligned}
& G_{q_{i} q_{j}}^{-1}=0 \text { for } q_{i} \neq q_{j} \text { and } q_{i}, q_{j} \neq r, \\
& G_{\varphi, r}^{-1}=G_{r, \varphi}^{-1}=0 .
\end{aligned}
$$

An analytical inversion of the matrix leads to the $\mathbf{G}$ matrix for the six-dimensional problem (given in Appendix A). The form obtained is unsuitable for the MCTDH propagation scheme, because it cannot be factorized as a sum of products of one-coordinate functions. This expression has been simplified based on two approximations. First, all terms proportional to $m_{H} / m_{C}$ have been neglected, leading to the diagonal matrix

$$
\begin{aligned}
& \widetilde{G}_{\varphi \varphi}=\frac{2}{m_{H} R_{c h}^{2}} \frac{1}{\Delta\left(\vartheta_{l}, \vartheta_{r}, \alpha_{l}, \alpha_{r}\right)}, \\
& \widetilde{G}_{\vartheta_{r} \vartheta_{r}}=\frac{1}{2 m_{H} R_{c h}^{2}\left(\cos \frac{\alpha_{r}}{2}\right)^{2}}, \\
& \widetilde{G}_{\vartheta_{l} \vartheta_{l}}=\frac{1}{2 m_{H} R_{c h}^{2}\left(\cos \frac{\alpha_{l}}{2}\right)^{2}}, \\
& \widetilde{G}_{\alpha_{r} \alpha_{r}}=\frac{2}{m_{H} R_{c h}^{2}}, \\
& \widetilde{G}_{\alpha_{l} \alpha_{l}}=\frac{2}{m_{H} R_{c h}^{2}},
\end{aligned}
$$


TABLE I. Grid sizes and numbers of single-particle functions for the MCTDH wave-packet propagation. $N$ indicates the number of Fourier grid points, "range" the limits (in degrees for the angles and a.u. for $r$ ) of the grid and $n_{t}$ the number of single particle functions used for the propagation up to the time $t$ in fs. $n_{50}^{*}$ indicates the number of single particle functions for converged calculation of diabatic and adiabatic populations up to 50 fs.

\begin{tabular}{cccccccccccc}
\hline \hline Coordinate & $N$ & Range & $n_{50}^{*}$ & $n_{20}$ & $n_{40}$ & $n_{50}$ & $n_{60}$ & $n_{70}$ & $n_{80}$ & $n_{90}$ & $n_{100}$ \\
\hline$\varphi$ & 128 & {$[-180: 180]$} & 40 & 28 & 28 & 28 & 32 & 32 & 32 & 36 \\
$\vartheta_{l}$ & 64 & {$[-120: 120]$} & 7 & 4 & 4 & 4 & 4 & 4 & 4 & 4 \\
$\vartheta_{r}$ & 64 & {$[-120: 120]$} & 7 & 4 & 4 & 4 & 4 & 4 & 4 & 4 \\
$\alpha_{l}-\alpha_{0}$ & 32 & {$[-63: 52]$} & 3 & 3 & 3 & 4 & 4 & 4 & 4 & 4 \\
$\alpha_{r}-\alpha_{0}$ & 32 & {$[-63: 52]$} & 3 & 3 & 3 & 4 & 4 & 4 & 4 & 4 \\
$r-r_{0}$ & 64 & {$[-0.9: 1.7]$} & 8 & 5 & 8 & 9 & 9 & 10 & 11 & 11 & 12 \\
\multicolumn{2}{l}{ Basis-set size } & 423,360 & 60,480 & 96,768 & 193,536 & 221,184 & 245,760 & 260,336 & 304,128 & 518,400 \\
\hline \hline
\end{tabular}

$$
\widetilde{G}_{r r}=\frac{2}{m_{C}},
$$

with

$$
\begin{aligned}
\Delta\left(\vartheta_{l}, \vartheta_{r}, \alpha_{l}, \alpha_{r}\right)= & \left(\sin \frac{\alpha_{l}}{2}\right)^{2} \cos ^{2} \vartheta_{l}+\left(\sin \frac{\alpha_{r}}{2}\right)^{2} \cos ^{2} \vartheta_{r} \\
& +\sin ^{2} \vartheta_{l}+\sin ^{2} \vartheta_{r} .
\end{aligned}
$$

A further simplification has been achieved by restricting $\widetilde{G}_{\varphi \varphi}$ to its value at the initial geometry. This approximation is based on a numerical inspection of $\widetilde{G}_{\varphi \varphi}$ for the relevant range of variation of the coordinates. The volume element is derived from the fact that the scissors mode, $\alpha$, and the pyramidalization, $\vartheta$, behave like a polar and an azimuthal angle, respectively. In particular, for $\alpha=180^{\circ}$ the pyramidalization angle is not defined. The corresponding part of the Jacobian is included in the kinetic-energy operator. The final expression for the kinetic-energy operator is given by

$$
\begin{aligned}
-2 \hat{T}= & \frac{2}{m_{H} R_{c h}^{2}} \frac{1}{\Delta\left(0,0, \alpha_{0}, \alpha_{0}\right)} \frac{\partial^{2}}{\partial \varphi^{2}}+\frac{2}{m_{C}} \frac{\partial^{2}}{\partial r^{2}} \\
& +\frac{1}{2 m_{H} R_{c h}^{2}\left(\cos \frac{\alpha_{r}}{2}\right)^{2}} \frac{\partial^{2}}{\partial \vartheta_{r}^{2}} \\
& +\frac{2 m_{H} R_{c h}^{2}\left(\cos \frac{\alpha_{l}}{2}\right)^{2}}{\frac{2}{\partial \vartheta_{l}^{2}}} \\
& +\frac{1}{m_{H} R_{c h}^{2}} \frac{1}{\sqrt{\cos \frac{\alpha_{r}}{2}}} \frac{\partial}{\partial \alpha_{r}} \cos \frac{\alpha_{r}}{2} \frac{\partial}{\partial \alpha_{r}} \frac{1}{\sqrt{\cos \frac{\alpha_{r}}{2}}} \\
& +\frac{2}{m_{H} R_{c h}^{2}} \frac{1}{\sqrt{\cos \frac{\alpha_{l}}{2}}} \frac{\partial}{\partial \alpha_{l}} \cos \frac{\alpha_{l}}{2} \frac{\partial}{\partial \alpha_{l}} \frac{1}{\sqrt{\cos \frac{\alpha_{l}}{2}}} .
\end{aligned}
$$

\section{NUMERICAL TECHNIQUES FOR THE DYNAMICAL STUDY}

\section{A. Wave-packet propagation with MCTDH}

The MCTDH method ${ }^{23,24}$ has been employed to follow the time evolution of the six-dimensional wave packet on the three coupled potential-energy sheets, starting initially on the diabatic $V$ state.

In the MCTDH approach, an optimized set of timedependent basis functions, $\phi_{n_{i}}^{(i)}$, called single-particle functions, is employed for the description of the wave function:

$$
\begin{aligned}
& \psi\left(\varphi, \vartheta_{l}, \vartheta_{r}, \alpha_{l}, \alpha_{r}, r, t\right) \\
& =\sum_{n_{1}} \cdots \sum_{n_{6}} \sum_{\sigma=1}^{3} A_{n_{1} \cdots n_{6} \sigma}(t) \quad \phi_{n_{1}}^{(1)}(\varphi, t) \quad \phi_{n_{2}}^{(2)}\left(\vartheta_{l}, t\right) \\
& \times \phi_{n_{3}}^{(3)}\left(\vartheta_{r}, t\right) \quad \phi_{n_{4}}^{(4)}\left(\alpha_{l}, t\right) \quad \phi_{n_{5}}^{(5)}\left(\alpha_{r}, t\right) \quad \phi_{n_{6}}^{(6)}(r, t) \chi_{\sigma}^{(d)},
\end{aligned}
$$

where the $A_{n_{1} \cdots n_{6} \sigma}$ denote the time-dependent expansion coefficients. In Eq. (6), $\chi_{\sigma}^{(d)}, \sigma=1,2,3$, are the three diabatic electronic states $V_{\pi^{2}}, V_{\pi^{*} 2}$, and $V_{\pi \pi^{*}}$ as defined in paper I, with $\chi_{1}^{(d)}\left(\chi_{2}^{(d)}\right)$ being the lowest state at $\varphi=0(\varphi=\pi)$. The equations of motion are derived from the Dirac-Frenkel variational principle. ${ }^{23-25} \mathrm{~A}$ scheme constructed along the lines suggested by Beck and Meyer ${ }^{39}$ has been employed for time integration. For all six degrees of freedom, a Fourier basis set has been used for the representation of the timedependent single-particle functions. Depending on the propagation time and the observable in question, different sets of single-particle functions have been employed. Details of the wave-function representations used are given in Table I.

Due to the large barrier between the two potential minima at $\varphi=0$ and $\varphi=\pi$, an initial state localized around $\varphi=0$ has been employed. The initial wave packet has been obtained by imaginary time propagation on the (diabatic) electronic ground-state surface in the vicinity of $\varphi=0$. The $\pi \rightarrow \pi^{*}$ photoexcitation process is modeled within the Condon approximation, that is, the electronic ground-state vibrational wave function is transferred from the $N$ state to the diabatic $V$ state to model the photoexcitation.

One of the observables of interest is the autocorrelation function $c(t)$ which is given by ${ }^{40}$ 


$$
c(t)=\left\langle\psi(t=0) \mid e^{-i H t} \psi(t=0)\right\rangle=\left\langle\psi^{*}(t / 2) \mid \psi(t / 2)\right\rangle,
$$

since the wave packet at $t=0$ is real. The absorption spectrum is given by the Fourier transform of the autocorrelation function. ${ }^{40}$

To characterize the radiationless decay dynamics after photoexcitation, the evolution of the diabatic electronic population ${ }^{41}$ has been computed

$$
\begin{array}{r}
P_{\sigma}^{(d)}(t)=\left\langle\psi(t)\left|\hat{P}_{\sigma}^{(d)}\right| \psi(t)\right\rangle, \quad \hat{P}_{\sigma}^{(d)}=\left|\chi_{\sigma}^{(d)}\right\rangle\left\langle\chi_{\sigma}^{(d)}\right|, \\
\sigma=1,2,3 .
\end{array}
$$

Since, for a large part of the configuration space, the $V$ diabatic state $\chi_{3}$ is very similar to the adiabatic $S_{1}$ state (in $D_{2}$ symmetry, for example), the decrease of $P_{3}(t)$ is closely related to the characteristic time scale of the radiationless decay. However, a more rigorous analysis requires the calculation of the adiabatic populations. ${ }^{42,43}$

$$
\begin{array}{r}
P_{\sigma}^{(a)}(t)=\left\langle\psi(t)\left|\hat{P}_{\sigma}^{(a)}\right| \psi(t)\right\rangle, \quad \hat{P}_{\sigma}^{(a)}=\left|\chi_{\sigma}^{(a)}\right\rangle\left\langle\chi_{\sigma}^{(a)}\right|, \\
\sigma=1,2,3,
\end{array}
$$

where the $\chi_{\sigma}^{(a)}$ are the adiabatic states obtained by the diagonalization of the diabatic potential matrix for each grid point in the six-dimensional space

$$
\chi^{(a)}=\mathbf{U}\left(\varphi, \vartheta_{l}, \vartheta_{r}, \alpha_{l}, \alpha_{r}, r\right) \boldsymbol{\chi}^{(d)} .
$$

While the evaluation of the kinetic-energy and potential matrix elements within the MCTDH propagation is straightforward (both are sums of products of one-dimensional operators or functions), the calculation of the adiabatic populations requires the evaluation of full-dimensional integrals. For the evaluation of the adiabatic projector matrix elements, the CDVR (correlation DVR) scheme of Ref. 44 has been used.

\section{B. Convergence tests}

A systematic and thorough check of the convergence with respect to the definition and size of the MCTDH basis set has been performed. The major tests consist in increasing systematically the number of single-particle functions for each of the six coordinates, until the observable of interest is unaffected. This has been done in a consistent way, i.e., by varying again all numbers of single-particle functions each time one of these numbers was found to be too small, to insure that the correlation between the six coordinates is accurately represented. Two sensitive examples of the behavior of these extensive convergence tests are shown in Figs. 1 and 2 for two particularly important coordinates, torsion and pyramidalization.

Figure 1 displays the development of the adiabatic ground-state population as a function of the number $\left(n_{\varphi}\right)$ of single-particle functions taken for the torsion $\varphi$. In the particular test presented, it can been seen that 36 single-particle functions are sufficient to obtain the converged ground-state population up to $50 \mathrm{fs}$. For a propagation up to $70 \mathrm{fs}$, this basis-set size is insufficient. When the basis-set sizes for the other degrees of freedom are optimized, 40 single-particle functions are required for convergence up to $50 \mathrm{fs}$. A similar study is presented in Fig. 2, which illustrates the convergence of the adiabatic ground-state population probability as

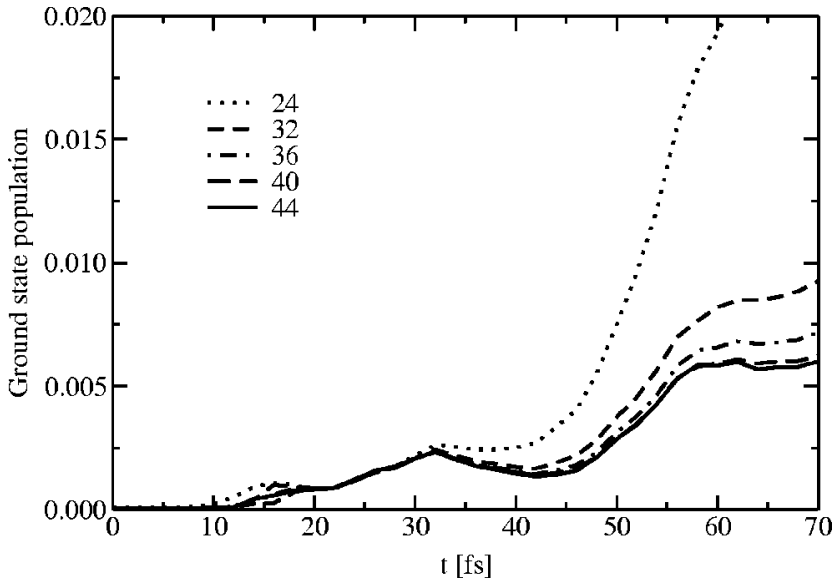

FIG. 1. Evolution of the population probability of the adiabatic ground state for different numbers of single-particle functions employed for the torsional coordinate $\varphi$.

a function of the number $\left(n_{\vartheta_{r}}=n_{\vartheta_{l}}\right)$ of single-particle functions taken for the pyramidalizations $\vartheta_{l}$ and $\vartheta_{r}$. It is important to realize that the effects of increasing $n_{\varphi}$ and $n_{\vartheta_{l}}$ $=n_{\vartheta_{r}}$ on the adiabatic population are opposite. An increase of $n_{\varphi}$ lowers the ground-state adiabatic population, whereas an increase of $n_{\vartheta_{l}}$ and $n_{\vartheta_{r}}$ enlarges it. A simultaneous increase of the basis sets for the torsion and for the pyramidalizations without a detailed investigation of the convergence with respect to the individual basis sets would be misleading, since the population probability would appear converged as a consequence of the compensation of the two effects.

The basis-set size required for convergence can depend significantly on the observables under consideration. Investigating either diabatic populations or adiabatic populations, slight differences in the number of necessary single-particle functions are found. The final basis set used for the computation of converged diabatic and adiabatic populations up to $50 \mathrm{fs}$ is given in Table I. It involves 423,360 Hartree products constructed from variationally determined time-dependent single-particle basis functions. Due to the high computational cost, simultaneous convergence of diabatic and adia-

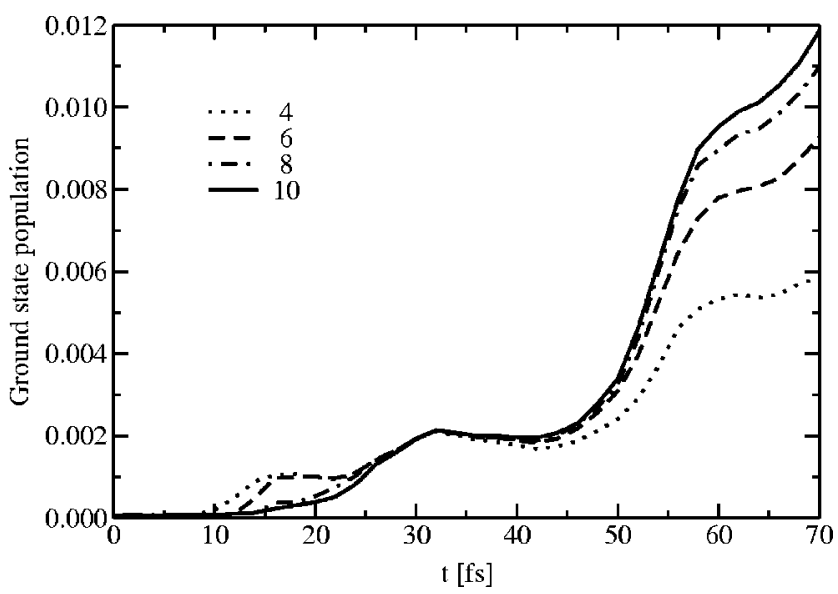

FIG. 2. Evolution of the population probability of the adiabatic ground state for different numbers of single-particle functions employed for the pyramidalization coordinates $\vartheta_{r}$ and $\vartheta_{l}$. 


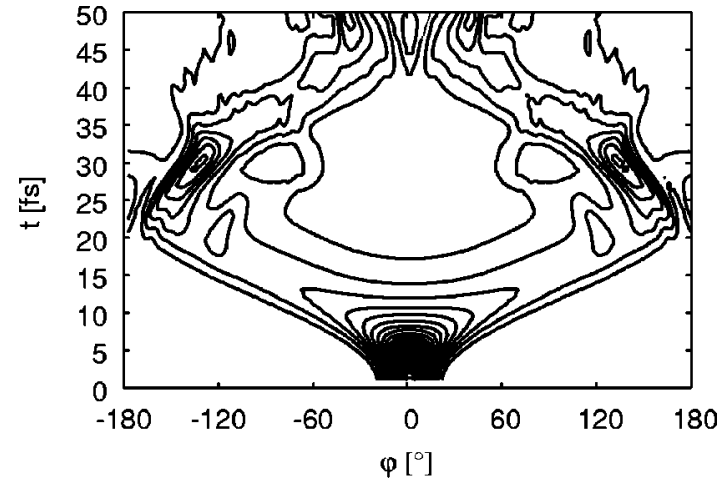

FIG. 3. Projected probability as a function of the torsional coordinate $\varphi$ and time.

batic populations has been attempted only for times up to 50 fs. A separate series of calculations has been performed to obtain converged diabatic populations up to $100 \mathrm{fs}$. The corresponding basis set, optimized for different time slots, is also given in Table I.

\section{RESULTS}

\section{A. Wave-packet evolution}

The time evolution of the wave packet prepared by instantaneous excitation from the $N$ state to the diabatic $V$ state has been studied by calculations of the projected probabilities along each of the six coordinates. The projected probability for mode $q_{i}$ is defined as the integral of the absolute square of the nuclear wave function over all remaining nuclear coordinates ${ }^{43}$ and summed over all electronic states. Figures 3-6 display 2D representations of this time evolution along the $\varphi, r, \alpha_{r}$, and $\vartheta_{r}$ coordinates. In Fig. 3, the very rapid torsional spreading of the wave packet initially localized at the planar geometry $(\varphi=0)$ can be observed. It takes $\simeq 10 \mathrm{fs}$ for the wave packet to reach the twist angle $\varphi=82^{\circ}$, where the first conical intersection is localized. ${ }^{19} \mathrm{In}$ $20 \mathrm{fs}$, the wave packet has completely spread and reaches $|\varphi|=\pi$. At longer times, the probability occupies the full range of $\varphi$ and becomes more structured, which indicates that a large amount of energy is available for the torsional motion. The representation of this structured wave packet requires a large number of single-particle functions. The evo-

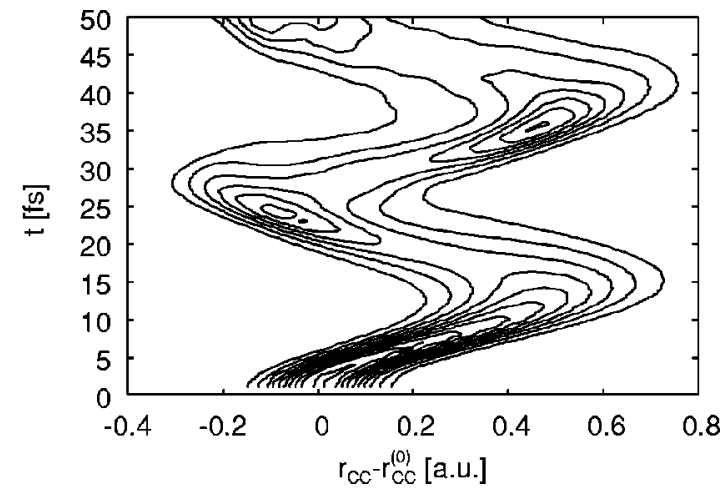

FIG. 4. Projected probability as a function of the CC stretching coordinate $r$ and time.

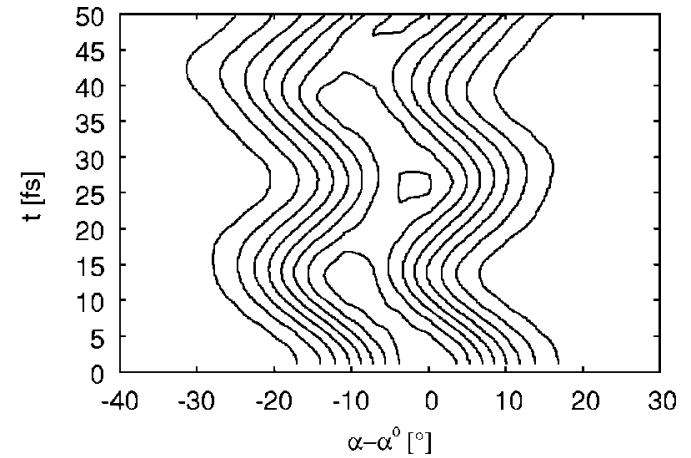

FIG. 5. Projected probability as a function of the scissor coordinates and time.

lution along the stretch coordinate $r$, presented in Fig. 4, is as expected for a $\pi \rightarrow \pi^{*}$ excitation process with a considerable elongation of the $\mathrm{CC}$ bond. The wave packet shows a quasiperiodic oscillatory motion with a frequency of $\simeq 25-30 \mathrm{fs}$. After $50 \mathrm{fs}$, the wave packet has broadened, but is still in coherent motion. Figure 5 shows the evolution along one of the scissors coordinates. The maximum of the projected probability oscillates only slightly both in amplitude and position, reaching a reduction of the $\mathrm{CH}_{2}$ angle by $10^{\circ}$. The evolution along one of the pyramidalization coordinates, displayed in Fig. 6, is characterized by a very limited motion of the wave packet. Only a beating of the width and a slow spreading of the wave packet are noticeable. After 50 fs, any sizeable fraction of the wave packet is still far away from the conical intersection situated at $\vartheta_{r} \simeq 110^{\circ} .{ }^{19}$

\section{B. Autocorrelation function and absorption spectrum}

The autocorrelation function shown in Fig. 7 decays in less than 5 fs. Test calculations (see below) have shown that both the torsional motion and the CC stretching motion contribute to this ultrafast initial decay of the autocorrelation function. A recurrence can be seen a little bit before $50 \mathrm{fs}$. The fast decay, which is responsible of the large overall width of the absorption spectrum, is similar to that obtained by Martínez and collaborators. ${ }^{30,45}$ However, the recurrence found in the present work occurs later than the recurrences they observed at $42 \mathrm{fs}^{30}$ and $33 \mathrm{fs},{ }^{45}$ depending on the level of the ab initio method used. Inspection of Figs. 3 and 4

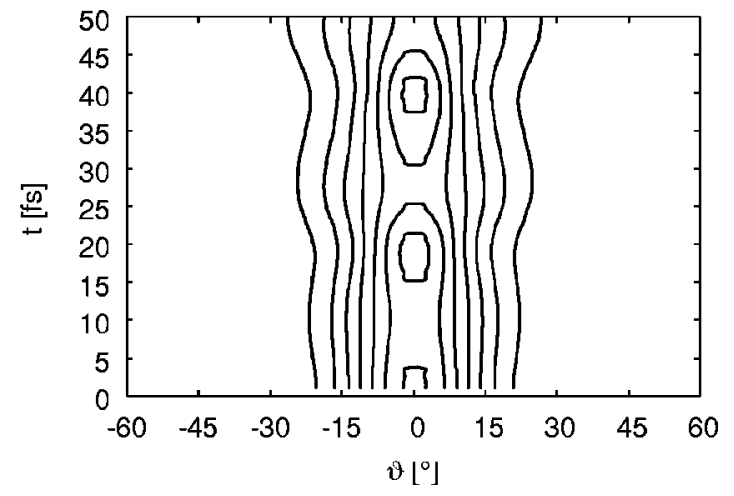

FIG. 6. Projected probability as a function of the pyramidalization coordinates and time. 


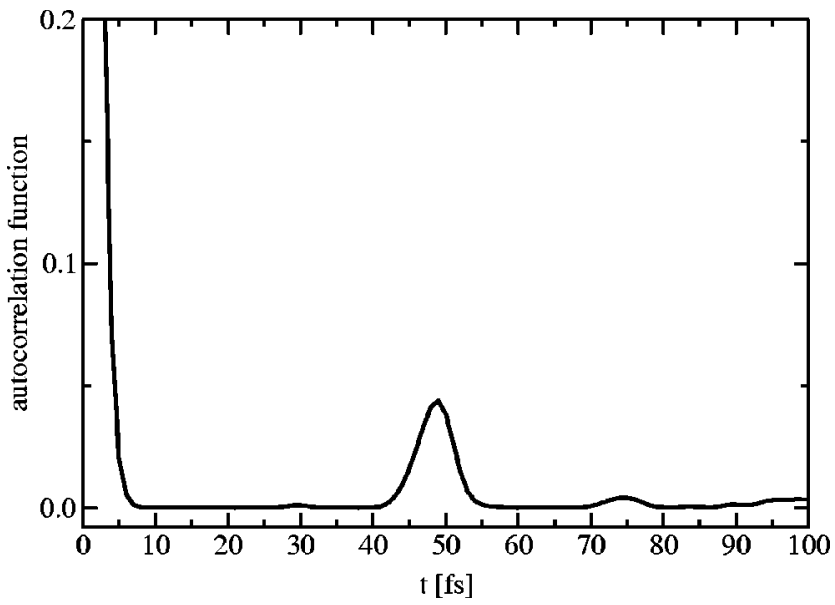

FIG. 7. Absolute value of the autocorrelation function $|c(t)|$ as a function of time. $|c(t)|$ is unity at $t=0$ and decays on a time scale of $5 \mathrm{fs}$. The vertical scale has been enlarged to reveal more clearly the weak recurrences near 30 , 50 , and 75 fs.

allows us to assign the prominent peak in the autocorrelation function to a combined recurrence of torsion and $\mathrm{CC}$ stretch motions, since around 50 fs the wave packet is again localized at $\varphi \sim 0$ and $r-r^{(0)} \sim 0$. From the inspection of the evolution of the projected probability along $r$ alone, one would expect a recurrence at around $25 \mathrm{fs}$, which is not seen. This behavior is quite different from that described in Refs. 30 and 45 where the recurrence is considered to be due to the stretch motion only.

To confirm this, three test calculations have been performed which include (i) only the torsional mode, (ii) only the stretching mode, and (iii) both of them, freezing all other modes at the ground-state equilibrium geometry. The corresponding autocorrelation functions are shown in Fig. 8. The calculation with the stretching coordinate only leads to an essentially periodic autocorrelation function with recurrences at multiples of $30 \mathrm{fs}$. The calculation for the torsional angle alone yields a more complicated autocorrelation function, reflecting the pronounced anharmonicity of the torsional PE function. The main recurrence occurs at $60 \mathrm{fs}$. The product of these two autocorrelation functions is notably different from the result of the two-dimensional calculation presented in Fig. 8(c), illustrating the nonseparability of these two modes. Addition of the four other nuclear degrees of freedom does not significantly modify the autocorrelation function for times smaller than $70 \mathrm{fs}$, except a reduction in the amplitude of the main recurrence. At longer times, a more pronounced damping of the recurrences is observed. Upon a closer look at the autocorrelation function of the six-dimensional calculation, two other recurrences with a much smaller amplitude can be detected around $30 \mathrm{fs}$ and $75 \mathrm{fs}$. The first one can be understood by the inspection of the wave-packet probabilities along $\varphi$ (Fig. 3), where a small spreading toward the initial wave-packet location $(\varphi=0)$ is observed. This broadening occurs at a time when the overlap of the wave packet with its initial form along $r$ is favorable (see Fig. 4), thus leading to a tiny recurrence in the autocorrelation function.

The corresponding spectrum, obtained as the Fourier transform of the autocorrelation function, is given in Fig. 9.

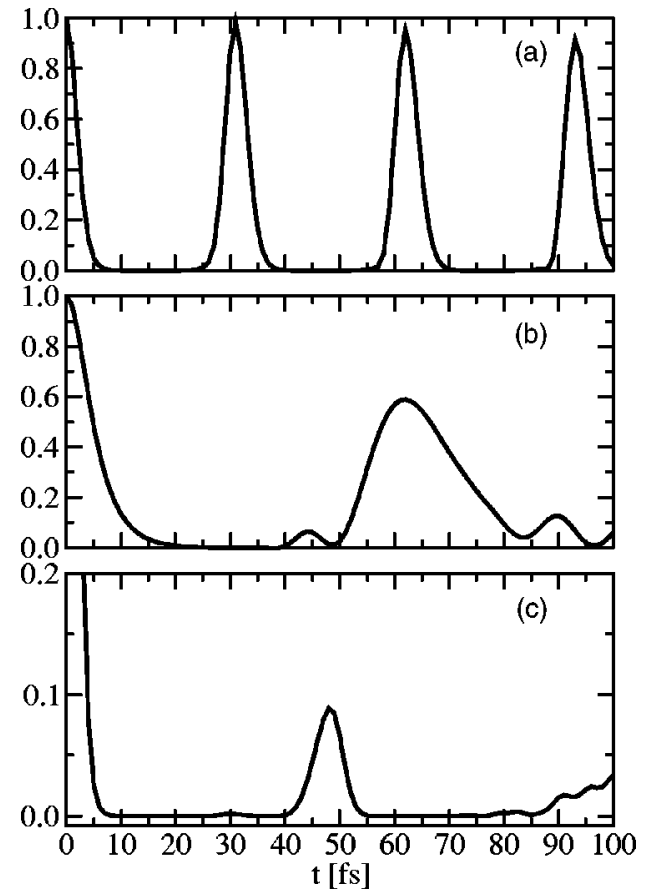

FIG. 8. Absolute value of the autocorrelation function $|c(t)|$ as a function of time for reduced dimensionality calculations considering (a) stretching coordinate only, (b) torsional mode only, and (c) stretching and torsional modes (note the different ordinate scales).

To remove spurious oscillations due to the finite propagation time, a Gaussian damping factor, $\exp \left(-t^{2} / t_{\text {damp }}^{2}\right)$ with $t_{\text {damp }}$ $=100 \mathrm{fs}$, has been employed. A direct comparison with the experimental spectrum ${ }^{2-4,36}$ is not easy, since the latter is overlaid by the absorption of Rydberg states which are not included in our model. In the experiment, the $\pi \rightarrow \pi^{*}$ excitation spectrum appears as a broad, weakly structured, underlying continuum. The spectrum has been theoretically simulated with both time-independent ${ }^{46,47}$ and time-dependent ${ }^{30,45}$ techniques. The overall shape of the spectrum obtained in the present work is similar to that of the previously published theoretical spectra. The peak structure is less regular on the low-energy side than on the high-energy side. These perturbations of the vibronic structure arise primarily from the strong anharmonic coupling of the torsional and stretching

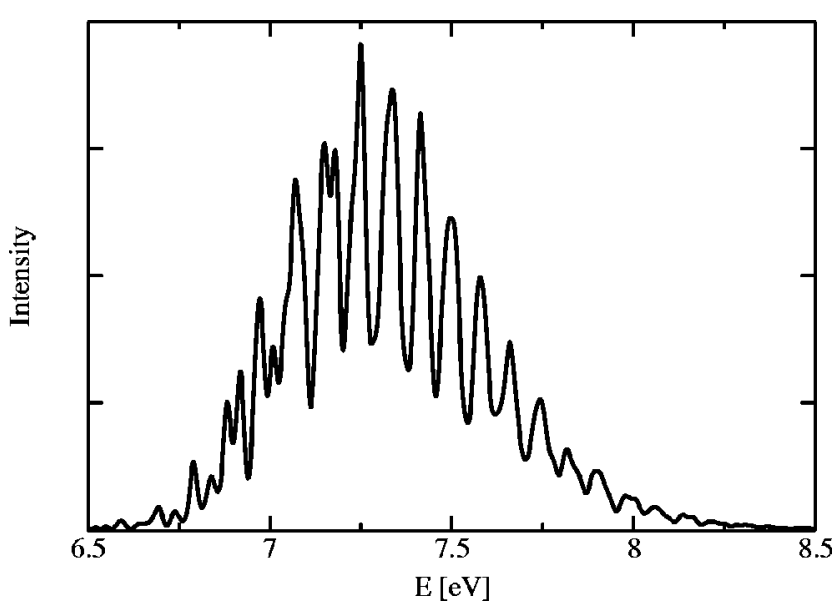

FIG. 9. Calculated absorption spectrum for $\pi \rightarrow \pi^{*}$ excitation of ethene. 


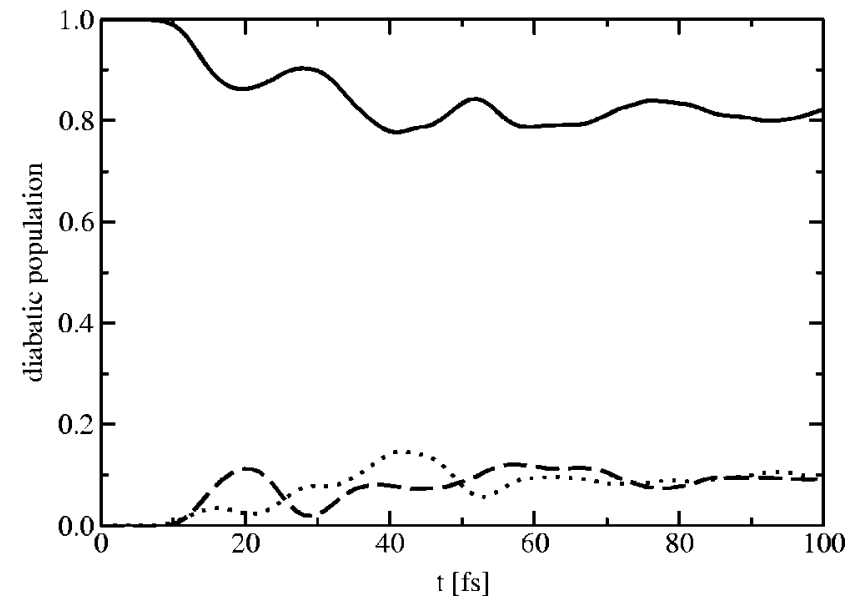

FIG. 10. Time evolution of the populations of the diabatic $V$ (full line), $N$ (dotted line), and $Z$ (dashed line) states.

modes. The peak spacing near the maximum of the absorption band is $680 \mathrm{~cm}^{-1}$, which is in reasonable agreement with the observed spacing of about $800 \mathrm{~cm}^{-1}$. 2,3 The modulation depth of the present spectrum is larger than observed in the experiment; this indicates that a broadening (i.e., optical dephasing) mechanism exists which is not recovered by the present $a b$ initio wave-packet calculations.

\section{Electronic populations}

The time scale of the radiationless decay can be assessed by inspecting the evolution of the electronic populations. Figure 10 presents the population of the three diabatic states as a function of time. The population of the initially populated diabatic $V$ state stays constant for about $10 \mathrm{fs}$, before the first decrease is observed leading to a loss of about $15 \%$ of the population up to $20 \mathrm{fs}$. The $10 \mathrm{fs}$ correspond to the time the wave packet needs to reach the twisted $\left(\varphi=82^{\circ}\right)$ geometry (see Fig. 3) where the first conical intersection is located. The step structure with a periodicity of $\simeq 30 \mathrm{fs}$ indicates that the population dynamics is partly driven by coherent motion in the $\mathrm{CC}$ stretch coordinate. An oscillatory exchange of population is noticeable for the two other diabatic states $(N$ and $Z)$ after $10 \mathrm{fs}$. They each account for a population of $10 \%$ after $50 \mathrm{fs}$, whereas the population of the $V$ has dropped to $80 \%$.

The interpretative value of the diabatic state populations, especially for the $N$ and $Z$ states, is limited, because the adiabatic states are a mixture of them. Only the $S_{1}$ adiabatic state is equivalent to the diabatic $V$ state in $D_{2}$ symmetry, that is, when only torsion and CC stretch are involved. The adiabatic electronic populations thus have to be considered for a better understanding of the transfer of electronic population to the ground state.

Figure 11 displays the adiabatic populations up to $50 \mathrm{fs}$. The evolution of the population of the adiabatic $S_{1}$ state is found to be very similar to that of the diabatic $V$ state. It stays close to unity for about $10 \mathrm{fs}$ and then decreases in a stepwise manner with a quasiperiodicity of $20-30 \mathrm{fs}$. It is seen that in addition to the stepwise decay driven by the CC stretching mode, there is a more gradual backflow of popu-

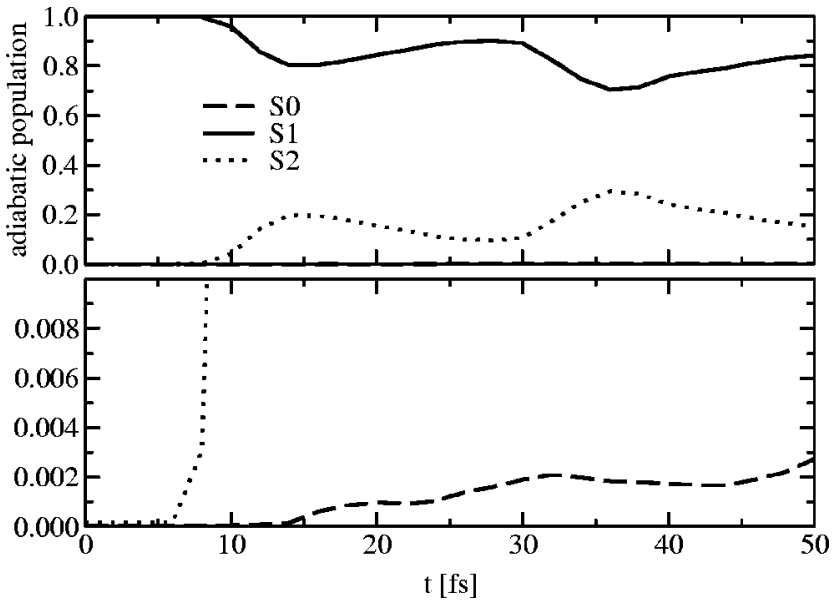

FIG. 11. Time evolution of the populations of the $S_{0}, S_{1}$, and $S_{2}$ adiabatic electronic states. In the lower panel, the scale has been enlarged to reveal the evolution of the ground-state population (dashed line).

lation to the $S_{1}$ state. The resulting overall decay is rather slow. At $10 \mathrm{fs}$, some population is found in the $S_{2}$ state, whereas the population of the ground state is still very small. Upon expansion of the scale (lower panel of Fig. 11), it can be seen that the transfer of population to the ground state starts at $15 \mathrm{fs}$. After $50 \mathrm{fs}$, the population in the ground state is only of $0.3 \%$. From these results as well as from the inspection of the time-dependent projected probabilities (especially along the pyramidalization coordinate), it is obvious that the wave packet does not find the conical intersection between $S_{1}$ and $S_{0}$ on the time scale of $50 \mathrm{fs}$. A closer look at the potential-energy surface ${ }^{19}$ reveals that the potential along the pyramidalization coordinate does not favor pyramidalization of twisted ethylene.

The influence of the scissors modes on the decay dynamics of the $V$ state has been assessed by a test calculation in which the two scissors modes have been frozen at the equilibrium geometry. The diabatic population of the $V$ state obtained in this four-dimensional calculation is shown in Fig. 12 together with the population obtained in the sixdimensional calculation. It is seen that the initial decay of the $V$-state population is significantly faster when the scissors

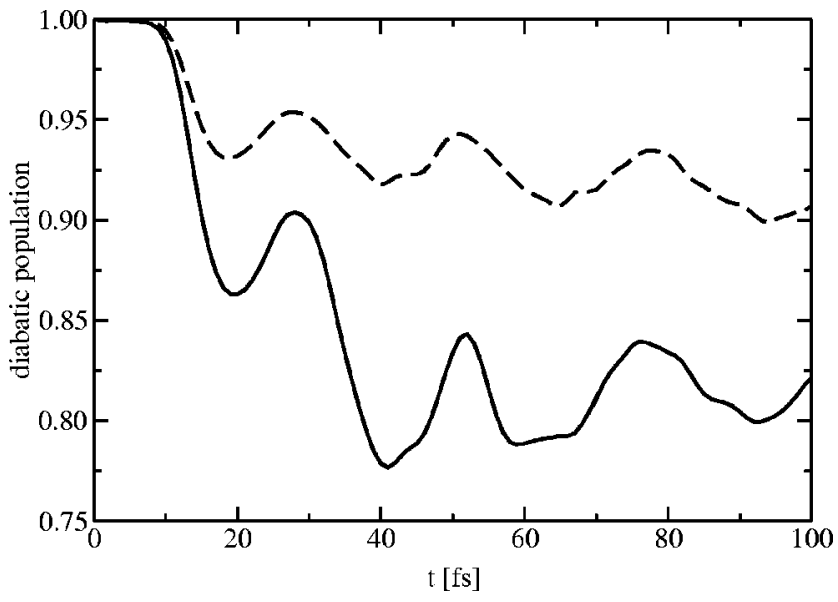

FIG. 12. Population probability of the diabatic $V$ state obtained with (full line) and without (dashed line) the scissors modes. 
modes are included. This is a direct consequence of the fact that the antisymmetric scissors mode couples the $V$ and $Z$ states in first order. ${ }^{12,19}$

A propagation for longer times with strict convergence criteria for both adiabatic and diabatic populations was found to be too time consuming to be performed. Nevertheless, the converged population of the diabatic $V$ state shown in Fig. 10 can be taken as an indication of the evolution of the population for the adiabatic $S_{1}$ state. It does not show a significant decay within the 100 fs of propagation. The oscillatory structure in the $V$ population is found to die out with time, but the $V$-state population remains on the order of $80 \%$ after 100 fs.

\section{DISCUSSION AND CONCLUSIONS}

Time-dependent quantum wave-packet calculations have been performed on a recently developed ${ }^{19}$ six-dimensional PE surface of the three valence states of ethene. The results provide new insight into the interpretation of the UV absorption spectrum and the radiationless decay dynamics of the $\pi \pi^{*}$ state of ethene.

As expected from numerous previous investigations, the torsion and the $\mathrm{CC}$ stretching vibration are found to be the dominant large-amplitude motions after Franck-Condon excitation of the $V$ state. The present multidimensional wavepacket calculations illustrate the pronounced anharmonic coupling of the torsional and CC stretch motions, which results from the (intuitively expected) strong dependence of the torsion potential on the CC distance. The analysis of the autocorrelation function reveals that the unusually large width of the $V$-state absorption spectrum arises from both the torsional and the CC stretching motions. The characteristic faint and extended vibrational progression in the UV absorption spectrum of ethene results from a weak recurrence of the autocorrelation function, which can neither be explained by one-dimensional torsion nor by one-dimensional CC stretch motion. The long-standing dispute about the assignment of the $V$-state vibrational structure ${ }^{2-5}$ appears pointless in the light of these results: the structure is not assignable in terms of uncoupled one-dimensional vibrational motions.

The primary intention of the present work has been the microscopic description of the radiationless decay dynamics of the $V$ state through the $V-Z$ and $Z-N$ conical intersections. As expected, ${ }^{9,10,14,17,18}$ the crossing (or near degeneracy) of the $V$ state with the $Z$ state at near-perpendicular geometry of ethene is reached by a fast spreading of the torsional wave packet on a time scale of the order of $10 \mathrm{fs}$. A new result of paper I and the present work is the finding that the antisymmetric scissors is the essential coupling mode for the $V-Z$ conical intersection. The antisymmetric scissors couples the $V$ and $Z$ states of perpendicular ethene in first order, whereas the pyramidalization can couple only in second order. ${ }^{19}$ The wave-packet calculations indeed show (see Fig. 12) that the linearly active asymmetric scissors mode leads to a significantly faster $V \rightarrow Z$ population transfer than the quadratically active pyramidalization.

The overall decay of the (adiabatic or diabatic) $V$-state population has been found to be rather slow, at least when compared with the predictions of previous direct-dynamics simulations, which have suggested population-decay lifetimes of $50 \mathrm{fs}^{32}$ and $180 \mathrm{fs},{ }^{31}$ respectively. The pyramidalization of perpendicular ethene has been identified as the dynamical bottleneck of the radiationless decay. The CASPT2 results of paper I indicate an initially very flat PE function for pyramidalization of perpendicular ethene, and even predict a small barrier. ${ }^{19}$ This result has been carefully checked $^{19}$ and is not considered to be an artifact of the CASPT2 calculations. Despite the considerable excess energy in the twisted $V$ state, the present quantum dynamics calculations predict a rather slow diffusive broadening of the probability density of the pyramidalization on a time scale of at least several hundred femtoseconds (see Fig. 6). The sluggish pyramidalization prevents the fast depopulation of the $Z$ state via the $Z-N$ conical intersection. Consequently, there is a backflow of $Z$-state population to the $V$ state (see Figs. 10 and 11), which is responsible for the overall rather slow decay of the photoexcited $V$ state.

The overall description of the photoinduced dynamics derived from the $a b$ initio multiple-spawning simulations of Ben-Nun and Martínez ${ }^{31}$ and the semiclassical directdynamics calculations of Granucci et $a l^{32}$ is at variance with the present results in so far as rapid pyramidalization and consequently rapid internal conversion to the electronic ground state have been predicted. Granucci et al. concluded that a large part of the $V$-state population is transferred to the ground state within $50 \mathrm{fs}$. In this simulation, the recrossing of trajectories from the ground state to the excited state has been suppressed, employing an energy criterion. This provision favors fast electronic decay. The calculations of BenNun and Martínez suggested a radiationless decay time of about $180 \mathrm{fs},{ }^{31}$ which is longer that the result of Ref. 32, but considerably faster than the $V$-state population decay time found in the present work.

In two recent femtosecond pump-probe experiments in the gas phase at $6 \mathrm{eV}$ excitation energy, a lifetime of 30 \pm 15 fs and $20 \pm 10$ fs after ${ }^{1} \pi \pi^{*}$ excitation of ethene has been measured. ${ }^{48,49}$ As discussed in Refs. 48 and 49 as well as in Ref. 30, this very short signal decay time most likely is not a population lifetime, but reflects the movement of the excited-state wave packet out of the detection window. The detection occurs via ionization to the ground state of the ethene cation. Since the equilibrium geometry of the cation is only weakly twisted, ${ }^{50}$ it is plausible that strongly twisted ethene molecules cannot be ionized with the probe pulses employed in the experiments. ${ }^{48,49}$ The measured lifetimes are indeed in good agreement with the calculated time scale of twisting of ethene in the $V$ state (cf. Fig. 3).

We do not feel called upon to comment on possible limitations of the direct-dynamics calculations, ${ }^{30-32}$ but we can discuss the possible sources of errors in the present quantum calculations. Apart from the approximation of the kineticenergy operator (see Appendix A), the calculations of this work are (numerically) exact for the given six-dimensional PE surface. Considering the ultrafast time scale and the fact that the qualitative signatures of the dynamics (rather that quantitative details) are of interest, the simplification of the kinetic-energy operator is not considered to be a relevant factor. The approximations inherent in the PE surface of re- 
duced dimensionality are more crucial and have been discussed in detail in paper I. Most critical is probably the freezing of six of the 12 internal coordinates of ethene. It is conceivable that a more effective reaction path to the $Z-N$ conical intersection seam exists when the $\mathrm{CH}$ stretch coordinates are allowed to adjust. ${ }^{16,38}$ The high-frequency $\mathrm{CH}$ stretching modes may also be of dynamical importance as accepting modes in the internal-conversion process. Alternatively, important inter-mode couplings may be lacking in the surface of paper I, although the torsion-pyramidalization coupling has been explicitly included. ${ }^{19}$ The accuracy of the CASPT2 data also should be checked in critical regions of the PE surface by more accurate MRCI calculations. ${ }^{8}$ Finally, nonadiabatic couplings with low-lying Rydbergs states, especially the $3 s$ Rydberg state, may play a role for the ra- diationless decay dynamics of the $V$ state (the Rydberg states have also been omitted, however, in the recent directdynamics simulations ${ }^{30,32}$ ). Clearly, more comprehensive and more accurate electronic-structure and dynamics calculations are required for a complete understanding of the photochemistry of the carbon-carbon double bond.

\section{ACKNOWLEDGMENTS}

A.V. acknowledges financial support by a Marie Curie fellowship of the European Community program Improving Human Research Potential and the Socio-Economic Knowledge Base under Contract No. HPMF-CT-2000-00840. U.M. and W.D. acknowledge financial support by the Deutsche Forschungsgemeinschaft and the Fonds der Chemischen Industrie.

\section{APPENDIX: KINETIC ENERGY OPERATOR}

The elements of the $\mathbf{G}$ matrix are given by

$$
\begin{aligned}
& G_{\varphi \varphi}=\frac{2}{m_{H} R_{c h}^{2}} \frac{1}{\Delta\left(\vartheta_{l}, \vartheta_{r}, \alpha_{l}, \alpha_{r}\right)}, \\
& G_{\vartheta_{r} \vartheta_{r}}=\frac{1}{2 m_{H} R_{c h}^{2}\left(\cos \frac{\alpha_{r}}{2}\right)^{2}} \frac{1}{1-\frac{m_{H}\left(\sin \frac{\alpha_{r}}{2}\right)^{2}}{m_{C}+m_{H}\left(2-\Delta\left(\vartheta_{l}, \vartheta_{r}, \alpha_{l}, \alpha_{r}\right)+\sin ^{2} \vartheta_{r}\right)}}, \\
& G_{\vartheta_{l} \vartheta_{l}}=\frac{1}{2 m_{H} R_{c h}^{2}\left(\cos \frac{\alpha_{l}}{2}\right)^{2}} \frac{1}{1-\frac{m_{H}\left(\sin \frac{\alpha_{l}}{2}\right)^{2}}{m_{C}+m_{H}\left(2-\Delta\left(\vartheta_{l}, \vartheta_{r}, \alpha_{l}, \alpha_{r}\right)+\sin ^{2} \vartheta_{l}\right)}},
\end{aligned}
$$

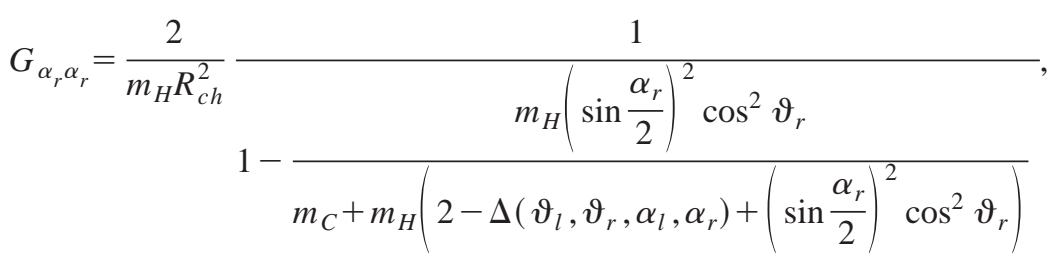

$$
\begin{aligned}
& G_{\alpha_{l} \alpha_{l}}=\frac{2}{m_{H} R_{c h}^{2}} \frac{1}{m_{H}\left(\sin \frac{\alpha_{l}}{2}\right)^{2} \cos ^{2} \vartheta_{l}}, \\
& 1-\overline{m_{C}+m_{H}\left(2-\Delta\left(\vartheta_{l}, \vartheta_{r}, \alpha_{l}, \alpha_{r}\right)+\left(\sin \frac{\alpha_{l}}{2}\right)^{2} \cos ^{2} \vartheta_{l}\right)} \\
& G_{r r}=\frac{2}{m_{C}+2 m_{H}-m_{H} \Delta\left(\vartheta_{l}, \vartheta_{r}, \alpha_{l}, \alpha_{r}\right)},
\end{aligned}
$$

with the following off-diagonal elements:

$$
G_{\vartheta_{r} \vartheta_{l}}=\frac{\sin \vartheta_{r} \sin \vartheta_{r}}{2 R_{c h}^{2} \cos \frac{\alpha_{l}}{2} \cos \frac{\alpha_{r}}{2}} \frac{1}{\left(m_{C}+2 m_{H}-m_{H} \Delta\left(\vartheta_{l}, \vartheta_{r}, \alpha_{l}, \alpha_{r}\right)\right)},
$$




$$
\begin{aligned}
& G_{\vartheta_{r} \alpha_{r}}=\frac{\cos \vartheta_{r} \sin \vartheta_{r} \tan \frac{\alpha_{r}}{2}}{R_{c h}^{2}} \frac{1}{\left(m_{C}+2 m_{H}-m_{H} \Delta\left(\vartheta_{l}, \vartheta_{r}, \alpha_{l}, \alpha_{r}\right)\right)}, \\
& G_{\vartheta_{r} \alpha_{l}}=\frac{\cos \vartheta_{l} \sin \vartheta_{r} \sin \frac{\alpha_{l}}{2}}{R_{c h}^{2} \cos \frac{\alpha_{r}}{2}} \frac{1}{\left(m_{C}+2 m_{H}-m_{H} \Delta\left(\vartheta_{l}, \vartheta_{r}, \alpha_{l}, \alpha_{r}\right)\right)}, \\
& G_{\vartheta_{r} r}=\frac{\sin \vartheta_{r}}{R_{c h} \cos \frac{\alpha_{r}}{2}} \frac{1}{\left(m_{C}+2 m_{H}-m_{H} \Delta\left(\vartheta_{l}, \vartheta_{r}, \alpha_{l}, \alpha_{r}\right)\right)}, \\
& G_{\vartheta_{l} \alpha_{r}}=\frac{\cos \vartheta_{r} \sin \vartheta_{l} \sin \frac{\alpha_{r}}{2}}{R_{c h}^{2} \cos \frac{\alpha_{l}}{2}} \frac{1}{\left(m_{C}+2 m_{H}-m_{H} \Delta\left(\vartheta_{l}, \vartheta_{r}, \alpha_{l}, \alpha_{r}\right)\right)}, \\
& G_{\vartheta_{l} \alpha_{l}}=\frac{\cos \vartheta_{l} \sin \vartheta_{l} \tan \frac{\alpha_{l}}{2}}{R_{c h}^{2}} \frac{1}{\left(m_{C}+2 m_{H}-m_{H} \Delta\left(\vartheta_{l}, \vartheta_{r}, \alpha_{l}, \alpha_{r}\right)\right)}, \\
& G_{\vartheta_{l} r}=\frac{\sin \vartheta_{l}}{R_{c h} \cos \frac{\alpha_{l}}{2}} \frac{1}{\left(m_{C}+2 m_{H}-m_{H} \Delta\left(\vartheta_{l}, \vartheta_{r}, \alpha_{l}, \alpha_{r}\right)\right)}, \\
& G_{\alpha_{r} \alpha_{l}}=\frac{2 \cos \vartheta_{l} \cos \vartheta_{r} \sin \frac{\alpha_{l}}{2} \sin \frac{\alpha_{r}}{2}}{R_{c h}^{2}} \frac{1}{\left(m_{C}+2 m_{H}-m_{H} \Delta\left(\vartheta_{l}, \vartheta_{r}, \alpha_{l}, \alpha_{r}\right)\right)}, \\
& G_{\alpha_{r} r}=\frac{2 \cos \vartheta_{r} \sin \frac{\alpha_{r}}{2}}{R_{c h}} \frac{1}{\left(m_{C}+2 m_{H}-m_{H} \Delta\left(\vartheta_{l}, \vartheta_{r}, \alpha_{l}, \alpha_{r}\right)\right)}, \\
& G_{\alpha_{l} r}=\frac{2 \cos \vartheta_{l} \sin \frac{\alpha_{l}}{2}}{R_{c h}} \frac{1}{\left(m_{C}+2 m_{H}-m_{H} \Delta\left(\vartheta_{l}, \vartheta_{r}, \alpha_{l}, \alpha_{r}\right)\right)},
\end{aligned}
$$

where

$$
\begin{aligned}
\Delta\left(\vartheta_{l}, \vartheta_{r}, \alpha_{l}, \alpha_{r}\right)= & \left(\sin \frac{\alpha_{l}}{2}\right)^{2} \cos ^{2} \vartheta_{l}+\left(\sin \frac{\alpha_{r}}{2}\right)^{2} \cos ^{2} \vartheta_{r} \\
& +\sin ^{2} \vartheta_{l}+\sin ^{2} \vartheta_{r}
\end{aligned}
$$

$$
\begin{aligned}
\widetilde{G}_{\vartheta_{l} \vartheta_{l}} & =\frac{1}{2 m_{H} R_{c h}^{2}\left(\cos \frac{\alpha_{l}}{2}\right)^{2}}, \\
\widetilde{G}_{\alpha_{r} \alpha_{r}} & =\frac{2}{m_{H} R_{c h}^{2}},
\end{aligned}
$$

We neglect all terms of order $m_{H} / m_{C}$, leading to the diagonal matrix

$$
\begin{aligned}
& \widetilde{G}_{\alpha_{l} \alpha_{l}}=\frac{2}{m_{H} R_{c h}^{2}}, \\
& \widetilde{G}_{r r}=\frac{2}{m_{C}} .
\end{aligned}
$$$$
\widetilde{G}_{\varphi \varphi}=\frac{2}{m_{H} R_{c h}^{2}} \frac{1}{\Delta\left(\vartheta_{l}, \vartheta_{r}, \alpha_{l}, \alpha_{r}\right)},
$$

$$
\widetilde{G}_{\vartheta_{r} \vartheta_{r}}=\frac{1}{2 m_{H} R_{c h}^{2}\left(\cos \frac{\alpha_{r}}{2}\right)^{2}},
$$

We further simplify the matrix by restricting $\widetilde{G}_{\varphi \varphi}$, which does not have a convenient form for MCTDH propagation, to its value at the initial geometry. 
${ }^{1}$ M. Klessinger and J. Michl, Excited States and Photochemistry of Organic Molecules (VCH, New York, 1995).

${ }^{2}$ A. J. Merer and R. S. Mulliken, Chem. Rev. 69, 639 (1969).

${ }^{3}$ M. B. Robin, Higher Excited States of Polyatomic Molecules (Academic, New York, 1975), Vol. 2, pp. 2-22.

${ }^{4}$ R. McDiarmid, J. Phys. Chem. 84, 64 (1980).

${ }^{5}$ R. McDiarmid, Adv. Chem. Phys. 110, 177 (1999).

${ }^{6}$ R. S. Mulliken, Phys. Rev. 48, 279 (1933).

${ }^{7}$ S. Krebs and R. J. Buenker, J. Chem. Phys. 106, 7208 (1997).

${ }^{8}$ T. Müller, M. Dallos, and H. Lischka, J. Chem. Phys. 110, 7176 (1999).

${ }^{9}$ R. J. Buenker, V. Bonačić-Koutecký, and L. Pogliani, J. Chem. Phys. 73, 1836 (1980).

${ }^{10}$ M. Persico and V. Bonačić-Koutecký, J. Chem. Phys. 76, 6018 (1982).

${ }^{11}$ V. Bonačić-Koutecký, P. Bruckmann, P. Hiberty, J. Koutecký, C. Leforestier, and L. Salem, Angew. Chem., Int. Ed. Engl. 14, 575 (1975).

${ }^{12}$ A. Viel, R. P. Krawczyk, U. Manthe, and W. Domcke, Angew. Chem., Int. Ed. Engl. 42, 3434 (2003).

${ }^{13}$ G. C. Groenenboom, Ph.D. thesis, TU Eindhoven, 1991.

${ }^{14}$ I. Ohmine, J. Chem. Phys. 83, 2348 (1985).

${ }^{15}$ E. M. Evleth and A. Sevin, J. Am. Chem. Soc. 103, 7414 (1981).

${ }^{16}$ L. Freund and M. Klessinger, Int. J. Quantum Chem. 70, 1023 (1998).

${ }^{17}$ M. Ben-Nun and T. J. Martínez, Chem. Phys. 259, 237 (2000).

${ }^{18}$ V. Molina, M. Merchán, B. O. Roos, and P.-A. Malmqvist, Phys. Chem. Chem. Phys. 2, 2211 (2000).

${ }^{19}$ R. P. Krawczyk, A. Viel, U. Manthe, and W. Domcke, J. Chem. Phys. 119, 1397 (2003).

${ }^{20}$ B. O. Roos, Adv. Chem. Phys. 69, 399 (1987).

${ }^{21}$ K. Andersson and B. O. Roos, in Modern Electronic Structure Theory, edited by D. R. Yarkony (World Scientific, Singapore, 1995), Vol. 1, pp. 55-109.

${ }^{22}$ T. H. Dunning, J. Chem. Phys. 90, 1007 (1989).

${ }^{23}$ H. D. Meyer, U. Manthe, and L. S. Cederbaum, Chem. Phys. Lett. 165, 73 (1990).

${ }^{24}$ U. Manthe, H. D. Meyer, and L. S. Cederbaum, J. Chem. Phys. 97, 3199 (1992).

${ }^{25}$ M. H. Beck, A. Jäckle, G. A. Worth, and H. D. Meyer, Phys. Rep. 324, 1 (2000).
${ }^{26}$ A. Raab, G. A. Worth, H. Meyer, and L. S. Cederbaum, J. Chem. Phys. 110, 936 (1999)

${ }^{27}$ S. Mahapatra, G. A. Worth, H.-D. Meyer, L. S. Cederbaum, and H. Köppel, J. Phys. Chem. A 150, 5567 (2001).

${ }^{28}$ C. Cattarius, G. A. Worth, H.-D. Meyer, and L. S. Cederbaum, J. Chem. Phys. 115, 2088 (2001).

${ }^{29}$ G. A. Worth, H.-D. Meyer, and L. S. Cederbaum, in Conical Intersections: Electronic Structure, Dynamics and Spectroscopy, edited by W. Domcke, D. R. Yarkony, and H. Köppel (World Scientific, Singapore, 2004), p. 583.

${ }^{30}$ M. Ben-Nun and T. J. Martínez, J. Phys. Chem. A 103, 10517 (1999).

${ }^{31}$ M. Ben-Nun and T. J. Martínez, Chem. Phys. Lett. 298, 57 (1998).

${ }^{32}$ G. Granucci, M. Persico, and A. Toniolo, J. Chem. Phys. 114, 10608 (2001).

${ }^{33}$ J. C. Tully, J. Chem. Phys. 93, 1061 (1990).

${ }^{34}$ G. Stock, J. Chem. Phys. 103, 1561 (1995).

${ }^{35}$ M. Ben-Nun and T. J. Martínez, J. Phys. Chem. A 104, 5161 (2000).

${ }^{36}$ R. J. Sension and B. S. Hudson, J. Chem. Phys. 90, 1377 (1989).

${ }^{37}$ B. A. Balko, J. Zhang, and Y. T. Lee, J. Chem. Phys. 97, 935 (1992).

${ }^{38}$ I. Ohmine, J. Chem. Phys. 83, 2348 (1985).

${ }^{39}$ M. H. Beck and H. D. Meyer, Z. Phys. D: At., Mol. Clusters 42, 113 (1997).

${ }^{40}$ E. J. Heller, Acc. Chem. Res. 14, 368 (1981).

${ }^{41}$ H. Köppel, W. Domcke, and L. S. Cederbaum, Adv. Chem. Phys. 57, 59 (1984).

${ }^{42}$ U. Manthe and H. Köppel, J. Chem. Phys. 93, 1658 (1990).

${ }^{43}$ W. Domcke and G. Stock, Adv. Chem. Phys. 100, 1 (1997).

${ }^{44}$ U. Manthe, J. Chem. Phys. 105, 6989 (1996).

${ }^{45}$ K. K. Baeck and T. J. Martínez, Chem. Phys. Lett. 375, 299 (2003).

${ }^{46}$ W. Siebrand, F. Zerbetto, and M. Z. Zgierski, Chem. Phys. Lett. 174, 119 (1990).

${ }^{47}$ A. M. Mebel, Y. T. Chen, and S. H. Lin, J. Chem. Phys. 105, 9007 (1996).

${ }^{48}$ P. Farmanara, V. Stert, and W. Radloff, Chem. Phys. Lett. 288, 518 (1998).

${ }^{49}$ J. M. Mestagh, J. P. Visticot, M. Elhanine, and B. Soep, J. Chem. Phys. 113, 237 (2000)

${ }^{50}$ H. Köppel, W. Domcke, L. S. Cederbaum, and W. Von Niessen, J. Chem. Phys. 69, 4252 (1978). 